\title{
Prevalence of coeliac disease among adult patients with autoimmune hypothyroidism in Jordan
}

\author{
O.H. Farahid, ${ }^{1}$ N. Khawaja, ${ }^{7}$ M.M. Shennak, ${ }^{2}$ A. Batieha, ${ }^{3}$ M. El-Khateeb ${ }^{1}$ and K. Ajlouni ${ }^{1}$
}



ABSTRACT The prevalence of coeliac disease among patients with autoimmune hypothyroidism has not been studied before in Jordan and other Arab countries. A cross-sectional record-based review was made of all adult autoimmune hypothyroidism patients who attended a referral centre in Jordan, during an 8-month period. Coeliac disease in these patients was diagnosed by the attending physician based on positive serological tests for anti-endomysial antibodies IgA and IgG followed by duodenal biopsy to confirm the diagnosis of coeliac disease. Of 914 patients recruited, 117 (12.8\%) were seropositive for coeliac disease. Of 87 seropositive patients who underwent duodenal biopsy, 39 had positive histological findings of coeliac disease (44.8\%). Extrapolating from these findings the overall rate of coeliac disease among autoimmune hypothyroidism patients was estimated to be $5.7 \%$. In multivariate logistic regression coeliac disease was significantly associated with older age ( $>40$ years), presence of other autoimmune diseases, vitamin B12 deficiency and anaemia.

Prévalence de la maladie cœliaque chez des patients adultes atteints d'hypothyroïdie auto-immune en Jordanie

RÉSUMÉ La prévalence de la maladie coeliaque chez des patients atteints d'hypothyrö̈die auto-immune n'a pas été étudiée auparavant en Jordanie, ni dans d'autres pays arabes. Un examen transversal à partir des dossiers de tous les patients adultes atteints d'hypothyrö̈die auto-immune qui avaient consulté un centre d'orientation en Jordanie au cours d'une période de huit mois a été mené. Le diagnostic de maladie cœliaque de ces patients a été posé par le médecin traitant à partir des analyses sérologiques positives pour les anticorps anti-endomysium de classe IgA et lgG, puis d'une biopsie duodénale à visée de confirmation du diagnostic de maladie cœliaque. Sur 914 patients recrutés, 117 (12,8 \%) étaient séropositifs pour la maladie cœliaque. Sur 87 patients séropositifs chez qui une biopsie duodénale a été réalisée, 39 ont eu des résultats histologiques positifs pour la maladie cœliaque (44,8\%). En extrapolant à partir de ces résultats, le taux global de maladie cœliaque chez les patients atteints d'hypothyroïdie auto-immune a été estimé à 5,7\%. Dans une analyse de régression logistique multivariée, la maladie cœliaque était significativement associée à un âge plus avancé (plus de 40 ans), à la présence concomitante d'autres maladies auto-immunes, à une carence en vitamine B12 et à une anémie.

'National Centre for Diabetes, Endocrinology and Genetics, Amman, Jordan (Correspondence to K. Ajlouni: ajlouni@ju.edu.jo).

${ }^{2}$ Department of Gastroenterology and Liver Disease, Faculty of Medicine, University of Jordan, Amman, Jordan.

${ }^{3}$ Department of Community Medicine, Public Health and Family Medicine, Faculty of Medicine, Jordan University of Science and Technology, Irbid, Jordan.

Received: 03/05/12; accepted: 24/09/12 


\section{Introduction}

Coeliac disease (gluten-sensitive enteropathy) is a systemic autoimmune disorder characterized by inflammation of the small-bowel mucosa, villous atrophy and crypt hyperplasia due to exposure to gliadin fraction of wheat gluten, hordein in barley, secalin in rye and aveninin oats and others in genetically susceptible individuals [1,2]. Based on the presence of anti-endomysial antibody (EMA) and anti-tissue transglutaminase (anti-tTG) antibody, with sensitivity and specificity reaching above $95 \%$, the prevalence of coeliac disease has increased in subclinical cases and several risk groups over the past 30 years [3]. Coeliac disease is associated with many autoimmune endocrine disorders, particularly type 1 diabetes mellitus, autoimmune thyroid diseases and others $[4,5]$. In previous studies, coeliac disease was found to be more prevalent in patients with autoimmune hypothyroidism $(\mathrm{AIH})$ with a prevalence of $3.3 \%-4.8 \%$ [6] compared with $1 \%$ in the general population [7].

To the best of our knowledge, the prevalence of coeliac disease among patients with AIH has not been investigated before in Jordan or other Arab countries. The aim of this study was to assess the prevalence of coeliac disease in patients with AIH in Jordan and to identify patients at higher risk of the disease.

\section{Methods}

\section{Study population}

A cross-sectional study was conducted at the National Centre for Diabetes, Endocrinology and Genetics, Amman, Jordan. All adult patients (a total of 914) with AIH who attended the Centre during the period October 2009 through May 2010, were included in the study. Exclusion criteria were hypothyroidism post-thyroidectomy or post-radioactive ablation therapy, pregnancy and thyroid malignancy.

\section{Data collection}

\section{Clinical data}

The diagnosis of AIH was made by the attending physician based on elevated thyroid-stimulating hormone (TSH) levels with reduced thyroxine and positive thyroid antibodies (anti-thyroid peroxidase and/or anti-thyroglobulin auto-antibodies). The diagnosis of coeliac disease was also made by the attending physician based on seropositivity of anti-EMA immunoglobulin (Ig)A and IgG. Patients who were seropositive for coeliac disease were further subjected to duodenal biopsy to confirm the diagnosis [8]. An experienced pathologist evaluated the biopsy according to the Modified Marsh Classification [9]. All data were collected from the medical records.

\section{Laboratory measurement methods}

Anti-EMA IgA and IgG levels were measured by generic assays (Dahlewitz $\mathrm{GmbH}$ ), an enzyme immunoassay for the quantitative determination of $\operatorname{IgA}$ and $\operatorname{Ig} G$ autoantibodies to human EMA. TSH, anti-thyroid peroxidase and anti-thyroglobulin levels were measured by 3rd-generation system (Axysym, Abbot), a microparticle enzyme immunoassay for the quantitative determination of TSH in human serum or plasma. All other measurements were carried out using standard techniques.

\section{Ethical considerations}

The study protocol was approved by the ethics committee of the National Centre for Diabetes, Endocrinology and Genetics. All data were kept strictly confidential and used for scientific purposes only. The study carried no harm to the patients as all data were obtained from routine medical records with no identifying information

\section{Statistical analysis}

The percentage of AIH patients with seropositivity to coeliac disease markers was obtained as well as the percentage of histologically confirmed coeliac disease. For the purpose of obtaining the overall rate of histologically confirmed coeliac disease in AIH patients, seropositive AIH patients who were not subjected to duodenal biopsy were assumed to be similar to those who underwent biopsy with respect to the percentage of histologically confirmed coeliac disease. The chi-squared test was used to determine the association of coeliac disease seropositivity with different variables. Multivariate logistic regression analysis was used to determine factors associated with seropositivity after controlling for potential confounders. The magnitude of the associations were expressed as odds ratios (OR). A 2-tailed $P$-value of 0.05 was considered statistically significant.

\section{Results}

\section{Participants' characteristics}

This study included 914 patients with AIH (806 females and 108 males) aged 20-82 years with a mean age of 51.1 years [standard deviation (SD) 13.4)]. The demographic, clinical and other relevant characteristics of the study population are presented in Table 1. The mean duration of AIH was 5.1 (SD 4.8) years, with almost $11 \%$ of patients having long-standing hypothyroidism of more than 10 years. The mean body mass index (BMI) was 32.2 (SD 6.7) $\mathrm{kg} / \mathrm{m}^{2} ; 60.7 \%$ of the AIH patients were obese and $26.8 \%$ were overweight. Around $42.2 \%$ had type 2 diabetes mellitus, $1.6 \%$ type 1 diabetes mellitus, $28.4 \%$ anaemia, $22.1 \%$ vitamin B 12 deficiency, $67.9 \%$ vitamin $\mathrm{D}$ deficiency and $3.7 \%$ other autoimmune diseases. The mean thyroxine dose was 723 (SD 389) $\mu \mathrm{g} /$ week (Table 1).

\section{Prevalence of coeliac disease among adult patients with AlH}

The seroprevalence of coeliac disease was $12.8 \%$ ( 117 out of 914 patients).

Only 87 seropositive patients underwent duodenal biopsy, and of these 39 had positive histological findings 


\begin{tabular}{|c|c|c|}
\hline Variable & No. & $\%$ \\
\hline \multicolumn{3}{|l|}{ Age (years) } \\
\hline $20-40$ & 199 & 21.8 \\
\hline $41-60$ & 472 & 51.6 \\
\hline $61-82$ & 243 & 26.6 \\
\hline \multicolumn{3}{|l|}{ Sex } \\
\hline Female & 806 & 88.2 \\
\hline Male & 108 & 11.8 \\
\hline \multicolumn{3}{|l|}{$B M I\left(\mathrm{~kg} / \mathrm{m}^{2}\right)$} \\
\hline Normal $(<27)$ & 114 & 12.5 \\
\hline Overweight (27-29) & 245 & 26.8 \\
\hline Obese (30+) & 555 & 60.7 \\
\hline \multicolumn{3}{|l|}{ Duration of AIH (years) } \\
\hline$\leq 5$ & 597 & 65.3 \\
\hline $6-10$ & 210 & 23.0 \\
\hline$>10$ & 107 & 11.7 \\
\hline \multicolumn{3}{|l|}{ Anaemia } \\
\hline$Y_{e s}{ }^{a}$ & 260 & 28.4 \\
\hline No & 654 & 71.6 \\
\hline \multicolumn{3}{|c|}{ Thyroxine dose ( $\mu \mathrm{g} /$ week) } \\
\hline $350-650$ & 311 & 34.0 \\
\hline $651-1000$ & 504 & 55.1 \\
\hline$>1000$ & 99 & 10.8 \\
\hline \multicolumn{3}{|l|}{ Diabetes } \\
\hline Yes & 386 & 42.2 \\
\hline No & 528 & 57.8 \\
\hline \multicolumn{3}{|l|}{ Vitamin B12 } \\
\hline Deficiency ${ }^{b}$ & 202 & 22.1 \\
\hline Normal & 712 & 77.9 \\
\hline \multicolumn{3}{|l|}{ Vitamin D deficiency } \\
\hline $\mathrm{Yes}^{\mathrm{c}}$ & 621 & 67.9 \\
\hline No & 293 & 32.1 \\
\hline \multicolumn{3}{|c|}{ Other autoimmune diseases } \\
\hline Present & 34 & 3.7 \\
\hline Absent & 880 & 96.3 \\
\hline
\end{tabular}

${ }^{a}$ Haemoglobin level $<13 \mathrm{~g} / \mathrm{dL}$ for males and $<12 \mathrm{~g} / \mathrm{dL}$ for females [16]; ${ }^{b}$ Vitamin B12 level $<208 \mathrm{pg} / \mathrm{mL}$; ${ }^{c 25}$ hydroxy vitamin D level $<30 \mathrm{ng} / \mathrm{mL}$.

of coeliac disease (44.8\%). The histological findings of coeliac disease were consistent with Marsh I in 5 (12.8\%) patients, Marsh II in 8 patients (20.8\%), Marsh IIIa in 12 patients (30.8\%), Marsh IIIb in 10 patients (25.6\%), and Marsh IIIc in 4 (10.3\%) patients with AIH.

Assuming that the remaining 30 seropositive AIH patients who were not subjected to duodenal biopsy had the same rate of coeliac disease as those who underwent duodenal biopsy, the overall rate of coeliac disease among AIH patients was estimated to be $5.7 \%$ (Table 2).

\section{Regression analysis}

Using multivariate logistic regression analysis it was found that anaemia (adjusted $\mathrm{OR}=3.7, P=0.04$ ), vitamin B12 deficiency (adjusted $\mathrm{OR}=3.0, P$ $=0.01$ ), and presence of other autoimmune diseases (adjusted $\mathrm{OR}=3.1$, $P=0.04)$ were significantly associated with coeliac disease (Table 2). In addition, older patients ( $>40$ years) were significantly 4.3 times more likely to have coeliac disease as compared with patients aged $20-40$ years $(P=$ $0.02)$. Each of these 4 variables were adjusted simultaneously for the other 3 variables. All other variables shown in Table 1, namely sex, BMI, duration of $\mathrm{AIH}$, thyroid dose, presence of diabetes and vitamin $\mathrm{D}$ deficiency were not independently related to coeliac disease seropositivity and were therefore removed from the logistic regression model.

\section{Autoimmune diseases in the study population}

A total of 34 patients had other associated autoimmune disorders, such as type 1 diabetes mellitus, Addison disease and vitiligo as shown in Table 3.

\section{Discussion}

The present study was the first in Jordan to assess the prevalence of coeliac disease in patients with AIH. The sample size we used was relatively large as compared with many previous studies conducted outside Jordan. Recent population screening studies have found that the prevalence of coeliac disease in Western countries approaches $1 \%$ [10]. The present study showed that the prevalence of histologically confirmed coeliac disease among patients with AIH was $5.7 \%$. This figure is slightly higher than the estimated range of global prevalence of coeliac disease among adult patients with $\mathrm{AIH}(3.3 \%-4.8 \%)$ [4]. The prevalences reported by Guilter et al. in a Turkish population [10], Berti et al. in Italians [11] and Hadithi et al. in Dutch patients [11] were $5.4 \%, 3.4 \%$ and $4.8 \%$ respectively. 


\begin{tabular}{|c|c|c|c|}
\hline Variable & OR & $95 \% \mathrm{Cl}$ & $P$-value \\
\hline Anaemia & & & 0.04 \\
\hline Yes & 3.7 & $0.63-16.9$ & \\
\hline $\mathrm{No}^{\mathrm{a}}$ & 1 & & \\
\hline Vitamin B12 & & & 0.01 \\
\hline Deficiency & 3.0 & $0.91-17.1$ & \\
\hline Normala $^{a}$ & 1 & & \\
\hline Other autoimmune diseases & & & 0.04 \\
\hline Present & 3.1 & $0.47-30.7$ & \\
\hline Absent $^{\mathrm{a}}$ & 1 & & \\
\hline Age years & & & 0.02 \\
\hline $20-40^{a}$ & 1 & & \\
\hline$>40$ & 4.3 & $1.04-28.1$ & \\
\hline
\end{tabular}

Each variable in the table was adjusted for all other variables in the table.

${ }^{a}$ Reference group.

$O R=$ odds ratio; $\mathrm{Cl}=$ confidence interval.
With respect to the seroprevalence of coeliac disease in patients with $\mathrm{AIH}$, several studies reported higher figures than that for histologically confirmed coeliac disease [11-13]. Hadithi et al. reported a seroprevalence of coeliac disease of $15 \%$ in patients with Hashimoto thyroiditis tTG [11], while Iuorio et al. reported a much higher coeliac disease seroprevalence of $27.4 \%$ using anti-EMA, both IgA and $\operatorname{IgG}[12]$. Our data showed a much lower seroprevalence of coeliac disease (12.8\%) than that reported by Iuorio et al. (27.5\%) [13]. However, Iuorio et al's study was based on a much smaller sample size (113 AIH patients) than our study (914 AIH patients).

In the present study, age $>40$ years, anaemia, vitamin B12 deficiency and using anti-gliadin, anti-EMA and anti- presence of other autoimmune diseases were independently associated with the seroprevalence of coeliac disease. Consistent with our findings, Ravaglia et al. reported significantly higher prevalence of coeliac disease in patients with $\mathrm{AIH}$ aged $\geq 65$ years than in patients aged $<65$ years $(P=0.02)$ [14]. Our data showed that AIH patients aged $>40$ years were 4.3 times more likely to have coeliac disease than patients aged $\leq 40$ years $(P=0.02)$.

With respect to anaemia, our findings of an association are consistent with the findings of Unsworth et al. who reported that $6 \%$ of 483 anaemic volunteers had coeliac disease [15], and Zamani et al. who reported that $14.6 \%$ of 206 anaemic patients had coeliac disease [16].

\begin{tabular}{lcc}
\hline $\begin{array}{l}\text { Table } 3 \text { Autoimmune diseases in the study group of patients with autoimmune } \\
\text { hypothyroidism }(\boldsymbol{n}=\mathbf{9 1 4})\end{array}$ & No. & $\%$ \\
Disease & 7 & 0.8 \\
Vitiligo & 8 & 0.9 \\
Addison disease & 2 & 0.2 \\
Systemic lupus erythematosus & 15 & 1.6 \\
Type 1 diabetes mellitus & 1 & 0.1 \\
Turner syndrome & 1 & 0.1 \\
Psoriasis & 880 & 96.3 \\
Normal &
\end{tabular}

Vitamin B12 deficiency is common in Jordan (48.1\%), as reported by Fora et al. [17], and this rate is higher than the reported global prevalence (ranging from $3 \%$ to $40 \%$ of the adult population) [18]. This difference is mainly related to ethnic variations and intestinal malabsorption problems [19]. To our knowledge, the association between vitamin B12 and coeliac disease had not been reported previously.

Our findings are consistent with a higher rate of coeliac disease in patients with other autoimmune diseases as had been previously reported by Kaukinen et al. [3]. Jiskra et al. found significantly higher serum levels of IgA anti-gliadin antibodies among 169 patients with autoimmune thyroid diseases, i.e. chronic thyroiditis and Graves' disease treated with a high replacement dosage of levothyroxine compared with patients treated with a lower dosage [14]. Our data, however, did not show an association between thyroxine replacement dosage and coeliac disease seroprevalence. This may be attributed to the use of anti-EMA antibodies in the present study, which have higher specificity and sensitivity than the IgA anti-gliadin antibodies used by Jiskara et al.

The present study had some limitations. First, it was a cross-sectional study, making it impossible to compare the prevalence of coeliac disease among patients with AIH and a control group. Secondly, we used only anti-EMA antibodies IgA and $\operatorname{IgG}$ as serological test for coeliac disease.

In conclusion, the prevalence of histologically-confirmed coeliac disease among patients with AIH in our study was estimated to be $5.7 \%$ compared with a seroprevalence of $12.8 \%$. Age > 40 years, anaemia, vitamin B12 deficiency and the presence of other autoimmune disorders were significantly associated with a higher seroprevalence of coeliac disease. These findings may provide a basis for screening of patients with $\mathrm{AIH}$ for coeliac disease, particularly the higher risk groups identified in this study. 


\section{References}

1. Green PH, Cellier C. Celiac disease. New England Journal of Medicine, 2007, 357:1731-1743.

2. Sanders DS et al. Changing face of adult coeliac disease: experience of a single university hospital in South Yorkshire. Postgraduate Medical Journal, 2002, 78:31-33.

3. Collin P et al. Antiendomysial and antihuman recombinant tissue transglutaminase antibodies in the diagnosis of coeliac disease: a biopsy-proven European multicentre study. European Journal of Gastroenterology and Hepatology, 2005, 17:85-91.

4. Kaukinen $\mathrm{K}$ et al. Celiac disease and autoimmune endocrinologic disorders. Digestive Diseases and Sciences, 1999, 44:1428-1433.

5. Collin P et al. Endocrinological disorders and celiac disease. Endocrine Reviews, 2002, 23:464-483.

6. Valentino $\mathrm{R}$ et al. Prevalence of coeliac disease in patients with thyroid autoimmunity. Hormone Research, 1999, 51:124-127.

7. West J et al. Seroprevalence, correlates, and characteristics of undetected coeliac disease in England. Gut, 2003, 52:960-965.

8. Green PH, Jabri B. Coeliac disease. Lancet, 2003, 362:383-391.

9. Oberhuber G, Granditsch G, Vogelsang H. The histopathology of coeliac disease: time for a standardized report scheme for pathologists. European Journal of Gastroenterology and Hepatology, 1999, 11:1185-1194.

10. Guliter $\mathrm{S}$ et al. Prevalence of coeliac disease in patients with autoimmune thyroiditis in a Turkish population. World Journal of Gastroenterology, 2007, 13:1599-1601.
11. Hadithi M et al. Coeliac disease in Dutch patients with Hashimoto's thyroiditis and vice versa. World Journal of Gastroenterology, 2007, 13:1715-1722.

12. luorio $\mathrm{R}$ et al. Prevalence of celiac disease in patients with autoimmune thyroiditis. Minerva Endocrinologica, 2007, 32:239243.

13. Jiskra $J$ et al. IgA and IgG antigliadin, IgA anti-tissue transglutaminase and antiendomysial antibodies in patients with autoimmune thyroid diseases and their relationship to thyroidal replacement therapy. Physiological Research, 2003, 52:79-88.

14. Ravaglia G et al. Increased prevalence of coeliac disease in autoimmune thyroiditis is restricted to aged patients. Experimental Gerontology, 2003, 38:589-595.

15. Unsworth DJ, Lock RJ, Harvey RF. Improving the diagnosis of coeliac disease in anaemic women. British Journal of Haematology, 2000, 111:898-901.

16. Zamani $\mathrm{F}$ et al. Gluten sensitive enteropathy in patients with iron deficiency anemia of obscure origin. World Journal of Gastroenterology, 2008, 14:7381-7385.

17. Fora MA, Mohammad MA. High frequency of suboptimal serum vitamin B12 level in adults in Jordan. Saudi Medical Journal, 2005, 26:1591-1595.

18. Stabler SP, Allen RH. Vitamin B12 deficiency as a worldwide problem. Annual Review of Nutrition, 2004, 24:299-326.

19. Gümürdülü $Y$ et al. Predictors of vitaminB12 deficiency: age and Helicobacter pylori load of antral mucosa. Turkish Journal of Gastroenterology, 2003, 14:44-49. 